\title{
The Transmit-Energy vs Computation-Delay Trade-off in Gateway-Selection for Heterogenous Cloud Aided Multi-UAV Systems
}

\author{
Ruiyang Duan, Student Member, IEEE, Jingjing Wang, Student Member, IEEE, \\ Chunxiao Jiang, Senior Member, IEEE, Yong Ren, Senior Member, IEEE \\ and Lajos Hanzo, Fellow, IEEE
}

\begin{abstract}
Unmanned aerial vehicles (UAVs) have been widely used in a range of compelling applications. In this paper, we integrate both the networking techniques and cloud computing tasks of multi-UAV systems. We commence by proposing an energy efficient scheme for selecting the gateway of UAVs invoked for relaying data to the heterogenous cloud. Then, relying on queuing theory and Lyapunov optimization, we strike a powerdelay trade-off by jointly optimizing the computational task scheduling and resource allocation in the heterogeneous cloud architecture, which is comprised of an edge cloud and a powerful remote cloud. We analyze the optimal resource-allocation strategy for each time slot and an iterative algorithm is conceived for reducing the computational complexity. Finally, our numerical results demonstrate the superiority of the proposed scheme.
\end{abstract}

Index Terms-Gateway selection, heterogenous cloud, powerdelay trade-off, task scheduling, resource allocation.

\section{INTRODUCTION}

G IVEN their low cost and high-flexibility deployment, unmanned aerial vehicles (UAVs) have been widely used both in military and in civilian applications for surveillance, environmental monitoring and emergency rescue, etc. Depending on their cruising duration and action radius, UAVs may be categorized into four classes, i.e. high-altitude and long-endurance UAVs, medium-range UAVs, short-range small UAVs and mini UAVs [1]. They are usually equipped with a variety of sensors in order to fulfill different tasks. Given the maturity of the UAV industry, small and mini UAVs have also been popularized among the public and their proliferation in diverse applications has attracted a lot of research attention. Recently, UAV communications have been

This work is supported by the National Natural Science Foundation of China (91338203), Young Elite Scientists Sponsorship Program By CAST (2016QNRC001), the Pre-research Fund of Equipments of Ministry of Education of China (6141A02022615), the EPSRC projects EP/Noo4558/1, EP/P34284, the ERC's Advanced Fellow Award QuantCom and the new strategic industries development projects of Shenzhen City (JCYJ20170816151922176). (Corresponding author: Chunxiao Jiang)

R. Duan, J. Wang and Y. Ren are with the Department of Electronic Engineering, Tsinghua University, Beijing, 100084, China. E-mail: duanry16@mails.tsinghua.edu.cn, chinaeephd@gmail.com, reny@tsinghua.edu.cn.

C. Jiang is with Tsinghua Space Center, Tsinghua University, Beijing, 100084; and also with the Research Institute of Tsinghua University in Shenzhen, Shenzhen 518057, China. E-mail: jchx@tsinghua.edu.cn.

L. Hanzo is with the school of Electronics and Computer Science, University of Southampton, Southampton, SO17, IBJ, U.K. Email: 1h@ecs.soton.ac.uk.

Copyright (c) 2012 IEEE. Personal use of this material is permitted. However, permission to use this material for any other purposes must be obtained from the IEEE by sending a request to pubs-permissions@iee.org. extensively studied for boosting the capacity and coverage of the existing wireless networks [2]-[6]. Specifically, UAVs can be used both as flying base stations and as relays as discussed in [3] and [6], respectively. The optimum altitudes of UAV for achieving the maximum capacity both in static and in mobile scenarios were derived in these contributions. A similar work considering the UAV's trajectory optimization at a fixed altitude was conducted by Zeng et al. in [4]. Moreover, UAVs have been introduced for Internet of Things (IoT) applications by Mozaffari et al. [5], where the UAVs are used for collecting data from IoT devices. Explicitly, the network association, the UAV placement and the devices' transmit power were jointly optimized for achieving maximum system capacity. However, their low load-carrying capacity and modest cruising capability have substantially limited the applications of small or mini UAVs. Additionally, computationally-intensive tasks impose challenges on these UAVs because of their limited processing capability and battery life. Hence, novel solutions should be conceived for enhancing the UAV's computational and communications capability.

Considering the limitations of a single UAV, the cooperation of multiple UAVs has been developed for improving the quality-of-service (QoS). The UAVs relying on sophisticated sensors can be coordinated by the ground station (GS) to fulfill specific tasks. The multi-UAV system concept was first proposed in [7] based on the flying ad hoc network (FANET) philosophy, which was later expanded in [8]-[10]. Although multi-UAV networks have substantial benefits over their single-UAV counterparts, they also have numerous challenges. Taking air-to-ground (A2G) communications as an example, if each UAV of the FANET is allowed to set up a communication link with the GS, they would lead to low spectral efficiency and severe interference. Hence, some of the superior drones should be chosen as the gateways to coordinate communications between the UAVs and the GS. Gateway selection schemes have been widely investigated in the context of mobile ad hoc networks (MANETs) [11]-[17]. In [12], Leng et al. proposed a k-hop compound metric based clustering scheme for selecting the gateways of a MANET, where the host connectivity and host mobility were jointly considered. Their simulation results showed that the scheme was characterized by rapid convergence despite its low control overhead. A network parameter optimization based gateway selection algorithm was proposed in [13] by Bouk et al. where 
multiple QoS parameters, such as the path availability period, the path's load capacity and latency were jointly optimized. Moreover, a fuzzy QoS balancing gateway selection algorithm was proposed by Zhioua et al. for vehicular networks [15], where the fuzzy logic was utilized for making decisions on the specific choice of the gateway relying on the received signal strength, on the traffic load of the cluster head, on the gateway candidates and on the link connectivity duration. They showed that the fuzzy scheme outperformed the deterministic scheme in terms of its adaptability. As for gateway selection in FANETs, Luo et al. [18] proposed a distributed gateway selection algorithm relying on the dynamic network partitioning concept, which considered the influence of the network topology on the gateway selection process. However, bearing in mind the high Doppler frequency, the limited energy resource and the constrained computational capability of FANETs, it is necessary to conceive a gateway selection scheme capable of balancing the QoS of all UAVs.

Mobile edge computing (MEC) and fog computing have become promising techniques for balancing and distributing the computationally-intensive tasks amongst resource-limited devices [19]-[21], since the devices can offload their tasks to cloud servers which are deployed locally in their vicinity, and the cloud servers return the final computational results to the devices. In [20], Bonomi et al. defined the characteristics of mobile edge/fog computing, which make it a suitable platform for both the IoT and big data analysis. The security and resilience of edge cloud were analysed by Shirazi et al in [21]. Relying on MEC and fog computing, both the power consumption and execution delay of the system can be substantially reduced. However, in comparison to traditional cloud computing, the computational resources in the edge cloud are typically restricted by its local configuration. Hence conceiving efficient resource allocation becomes a critical issue in MEC, which has therefore attracted much attention [22]-[28]. Specifically, in [22], Sardellitti et al. proposed an iterative algorithm based on successive convex approximation for jointly allocating both the radio resources and computational resources to multiple users in a multiple-input and multiple-output (MIMO) aided MEC system. Moreover, a power-vs-delay trade-off was formulated in [23] in the context of a multi-user MEC system, where the local processing capability of devices was considered and an optimal resource allocation scheme was designed with the aid of Lyapunov optimization. The power-vs-delay trade-off problems were also studied in [24], [25] with the Lyapunov optimization framework. In [26], Liu et al. studied the delay-optimal task scheduling and resource allocation problem under specific power constraints in MEC systems, where the optimal strategy was modeled by a Markov decision process. Their scheme was capable of achieving shorter average execution delay than their benchmark schemes. The computation offloading decision, the physical resource block allocation and the MEC computational resource allocation were integrated into an amalgamated framework and were jointly optimized in [28] by Wang et al., who achieved a better integrated performance than classic resource allocation schemes. However, the existing works are focused on the interplay between the devices and edge cloud, while ignoring the interaction between the edge cloud and the powerful remote cloud.

In order to further improve the QoS performance, relying on both the flexible configuration of the edge cloud and on the more powerful computational capability of the remote cloud, a beneficial architecture combining both the edge cloud and the remote cloud has been developed in [29]-[35]. To elaborate a little further, Gelenbe et al. [30] studied the optimal load sharing problem between a local and a remote cloud, where an optimal scheme was proposed based on the analysis of the power consumption and the computing time in the context of diverse tasks and requirements. The fairness of resource allocation problems was investigated in [32] in the heterogenous cloud context, where a multi-resource allocation mechanism was designed for guaranteeing fairness, whilst maintaining service isolation amongst the users. Moreover, the delay-bounded task offloading problem of heterogenous cloud based systems was highlighted by Zhao et al. [33] upon considering both the wireless transmission delay as well as the computational execution delay. They modeled the service arrival process by the classic $\mathrm{M} / \mathrm{M} / 1$ queue. Based on this model, the success probability of the delay-bounded task execution was derived both in the context of a single-user and a multi-user scenario. Finally, a total power minimization based task scheduling problem was studied by Gai et al. [35].

Against this background, in this paper, we focus on the combination of UAV networks, MEC and the heterogenous cloud. Specifically, we study the QoS-based network association problem, with a special focus on the gateway selection and resource allocation of our heterogenous cloud based multiUAV system. To elaborate, different UAVs jointly constitute a group in a FANET, and the computational tasks of the different FANETs can be offloaded to the heterogenous cloud composed of an edge cloud and a remote cloud. The heterogenous cloud platform then deals with these tasks and provides feedback to the control center. We consider a pair of QoS metrics, namely the energy/power consumption as well as the transmission and execution time delay, demonstrating that this framework significantly improves the system performance. We shed light on how the cloud interacts with UAVs and on how the cooperation between the clouds improves the system performance. Our main contributions are summarized as follows:

- By jointly optimizing both the energy consumption and the data transmission time, we conceive an energyefficient gateway selection scheme, where the specific UAV, which consumes the least energy can be selected as the gateway in each time slot.

- Relying on analyzing the power consumption and cloudbased computation execution delay, we formulate a power consumption minimization problem under strict system stability constraints in order to find the optimal taskscheduling and resource allocation strategy. Furthermore, a power-vs-delay trade-off is struck based on Lyapunov optimization [36] and an iterative algorithm is conceived for finding the optimal strategy.

- Our extensive simulations demonstrate the efficiency of our proposed algorithms, which are characterized by rapid convergence as well as by a beneficial power/energy 


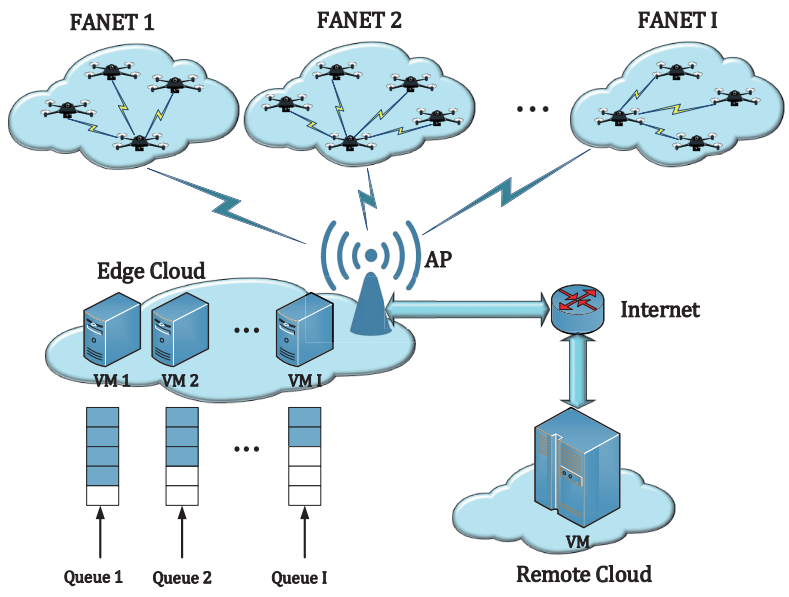

Fig. 1. The structure of the heterogeneous cloud-aided multi-UAV system.

consumption and transmission/execution delay.

The remainder of this paper is organized as follows. The system model, including the UAV model, the channel model and the cloud execution model, is introduced in Section II. In Section III, our energy efficient gateway selection scheme is characterized based on the analysis of the energy consumption and data transmission time. In Section IV, the power-vs-delay trade-off is improved based on Lyapunov optimization. Moreover, an iterative algorithm is invoked for finding the optimal strategy in each time slot. Finally, our simulation results are provided in Section $\mathrm{V}$ for evaluating the performance of our proposed scheme, followed by our conclusions in Section VI.

\section{System ModeL}

In this paper, we consider a heterogeneous cloud-based multi-UAV system, which consists of multiple UAV clusters, an edge cloud and a remote cloud, as shown in Fig. 1. The UAVs in different clusters carry sensed data related to different tasks, while the UAVs in the same cluster constitute a FANET. We assume that these tasks are computationally-intensive, hence the UAVs have to offload the tasks to the clouds for their execution. The UAVs are able to communicate with the clouds through a radio access network (RAN). In our model, the edge cloud can be viewed as a small processing center with limited computational capabilities at the wireless access point (AP), while the remote cloud is a large-scale processing center with powerful processing capabilities with the aid of its high-rate, low-delay Internet backbone. The system is operated under dynamically fluctuating conditions because of the high mobility of the UAV nodes. For the simplicity of analysis, the dynamic fluctuations of the system are discretized into time slots, i.e. $t \in \mathcal{T}=\{1,2,3, \ldots\}$. We assume that the topology of different UAV clusters remains relatively time-invariant in one slot. In each time slot, first a gateway is selected to relay the sensed data of different UAVs within the same FANET to the AP over the air, and then the data is processed by the virtualized machines (VMs) either within the edge cloud or in the remote cloud.

\section{A. The UAV Model}

We assume that there are $I$ FANETs executing $I$ tasks in the multi-UAV system, which are denoted as $\left\{\mathcal{G}_{1}, \mathcal{G}_{2}, \ldots, \mathcal{G}_{I}\right\}$, while $\mathcal{G}_{i}=\left[1,2, \ldots, N_{i}\right]$ represents all the UAVs in FANET $i$. The tasks are assigned by the GS. Once being assigned a task, the related UAVs will fly to certain area according to the pre-defined path, collect the related data, then offload the data to the nearest AP. These UAVs are equipped with sensor units, control units, management units and communication units to fulfill their tasks. Specifically, the communication units are composed of multiple modules configured by various protocols [1], so that the UAVs can communicate with the AP via Wi$\mathrm{Fi}$, long-term evolution (LTE) transceivers, etc. as required. We assume that the gateways are allocated orthogonal resources (e.g., OFDMA systems) so that the interferences between different gateways can be neglected and that the bandwidth allocated to each gateway is $B$. The UAVs in the same FANET communicate with each other using the IEEE protocol $802.11 \mathrm{~b} / \mathrm{g}$ (WiFi). Since the inter-FANET communication is beyond the focus of this paper, we assume that the energy consumption and the delay between UAVs and the gateway can be neglected compared to that caused by air-to-ground (A2G) communication and cloud-based computations. Under this assumption, we only consider the packet-arrivals from the selected gateway. Let $A_{i}(t)$ denote the number of packets arriving at the AP from the gateway of $\mathcal{G}_{i}$ in time slot $t$, while $\boldsymbol{A}(t)=\left[A_{1}(t), A_{2}(t), \ldots, A_{I}(t)\right]$ denotes the vector of arrivals in time slot $t$, where $A_{i}(t)$ in the different time slots is independent and identically distributed (i.i.d.). We also assume that the number of packet-arrivals is bounded by $A_{i, \min } \leq A_{i}(t) \leq A_{i, \max }$ and that $A_{i}(t)$ is uniformly distributed between $\left[A_{i, \min }, A_{i, \max }\right]$. Let $\lambda_{i}=E\left[A_{i}(t)\right]$ denote the rate of packet arrival from the gateway of $\mathcal{G}_{i}$ in time slot $t$.

\section{B. The Channel Model}

In contrast to cellular networks, in $\mathrm{A} 2 \mathrm{G}$ communications, each UAV typically has a line-of-sight (LoS) path towards the AP with a given probability. The probability of LoS propagation depends both on the environment and on the elevation angle, which may be quantified by [5] [37]:

$$
f^{\mathrm{LoS}}=\frac{1}{1+\phi \exp (-\varphi[\theta-\phi])},
$$

where $\phi$ and $\varphi$ are constants that depend on the specific environment and $\theta$ is the elevation angle. Let us denote the communication distance as $d$. Then $\theta$ is calculated by $\theta=\frac{180}{\pi} \times \sin ^{-1}\left(\frac{H}{d}\right)$, where $H$ is the hovering altitude of the UAV. Therefore, an averaged path loss model considering both the LoS and NLoS links having a Rician block-fading model is invoked for characterizing the channel between the UAV and the AP, where the Rician fading coefficient remains constant in each block, but changes randomly from one block to another. We assume that the block duration is the time needed to transmit a packet. Hence, the channel model is given by:

$$
h=\left(\xi_{1} f^{\mathrm{LoS}}+\xi_{2} f^{\mathrm{NLoS}}\right) d^{-\alpha / 2} \tilde{h},
$$


where $\xi_{1}$ and $\xi_{2}$ are path loss coefficients in the LoS and NLoS cases, $\alpha$ denotes the path loss exponent, $\tilde{h}$ represents the fast fading coefficient and $f^{\mathrm{NLoS}}=1-f^{\mathrm{LoS}}$. We consider the effect of path loss and fast fading, while the effect of shadowing is neglected. Moreover, in this article, the fast fading coefficient $\tilde{h}$ is formulated as:

$$
\tilde{h}=X_{1}+j X_{2},
$$

where $X_{1} \sim \mathcal{N}\left(\mu_{1}, \sigma^{2}\right)$ and $X_{2} \sim \mathcal{N}\left(\mu_{2}, \sigma^{2}\right)$ are Gaussian random variables. Therefore, the Rician probability density function (PDF) of $|\tilde{h}|$ is expressed as:

$$
f_{|\tilde{h}|}(z)=\frac{\sigma^{2}}{z} \exp \left[-\frac{1}{2 \sigma^{2}}\left(z^{2}+A^{2}\right)\right] I_{0}\left(\frac{A z}{\sigma^{2}}\right),
$$

where $A^{2}=\mu_{1}^{2}+\mu_{2}^{2}$ is the power of the LOS signal, while $I_{0}$ is the 0 -th order modified Bessel function of the first kind.

\section{Cloud Computation Model}

In our paper, the packets arriving from different FANETs are offloaded and processed in the clouds. Once data transmission from the gateways and the AP is finished, we assume that part of the tasks of FANET $i$, denoted as $A_{e, i}(t)$, will be processed in the edge cloud, while $A_{r, i}(t)$ packets of the tasks will be processed in the remote cloud. Here we have $A_{i}(t)=$ $A_{e, i}(t)+A_{r, i}(t)$.

1) Edge Cloud: In the edge cloud, there are a total of $I$ VMs exclusively used by $I$ FANETs. When the packets reach the edge cloud, the corresponding VM allocates appropriate computational resources to carry out the processing task. The computational resources may be quantified in terms of the number of CPU-cycles [23]. Naturally, the number of CPUcycles per unit time-duration is proportional to the CPU's clock-frequency. In this paper, we assume that FANET $i$ has $n_{i}(t)$ CPU-cycles in time slot $t$, while the total number of CPU-cycles per unit time in the edge cloud is $n_{\max }$. We express the number of packets that can be processed by the VM $i$ in the edge cloud as:

$$
\mu_{i}(t)=\Delta t n_{i}(t) L_{i}^{-1}, \quad \Delta t>n_{i}(t) L_{i}^{-1}
$$

where $\Delta t$ denotes the duration of the time slot and $L_{i}$ is the minimum number of CPU cycles needed to process a single packet received from FANET $i$. Moreover, the power consumption of the computations in the edge cloud can be approximated by [23]:

$$
P_{e, i}(t)=\kappa n_{i}^{3}(t)
$$

where $\kappa$ is the capacitance proportionality factor.

The newly arrived packets will be queued in the buffer of the edge cloud, when the corresponding VM is busy processing the earlier packets. Let $Q_{i}(t)$ denote the queue length in the $i$-th VM related to FANET $i$, where we have $Q_{i}(0)=0$ at the beginning. Then the evolution of the queue length can be expressed as:

$$
Q_{i}(t+1)=\max \left\{Q_{i}(t)-\mu_{i}(t), 0\right\}+A_{e, i}(t) .
$$

The computational delay of the edge cloud may simply arise from managing the queue. Relying on Little's Law [38], the average queueing delay can be derived from the average queue length, which can be calculated from Eq. (7).

2) Remote Cloud: By contrast, the power consumption in the remote cloud is closely related to the variation of the computational workload, which is formulated by:

$$
P_{r, i}(t)=C e^{A_{r, i}(t)}
$$

where $C$ is a proportionality coefficient. As for the execution delay in the remote cloud, given that it is equipped with a multi-core high-speed CPU, we assume that the packets arriving can be processed without any queueing delay. Hence, the time delay essentially depends on the Internet transmission process. Moreover, according to [39], the Internet's transmission delay can only be characterized empirically. For simplicity, we assume that the Internet's transmission process has a deterministic delay $D$.

\section{ENERGY-EFFICIENT GATEWAY SELECTION}

As mentioned in Section I, if each UAV in a FANET establishes a communication link with the AP, this may lead to low spectral efficiency and high interferences at the AP. Hence, it is necessary to select superior UAVs to act as gateways so that the ordinary UAVs in the FANET can communicate with the AP via the relay of gateway. In this section, we design an energyefficient gateway selection scheme considering both the energy and the time needed for data transmission. Given our stochastic channel, it may result in a probabilistic communication outage as well as stochastic energy consumption and time delay. Without loss of generality, in the following, we only consider FANET $i$ as a simple example and study how to select the gateway drone.

\section{A. The Communication Model Analysis}

In time slot $t$, let $u$ denote the UAV selected as the gateway in FANET $i$, where $u \in \mathcal{G}_{i}$. Then the signal received at the AP from $u$ can be modeled as:

$$
y=\sqrt{P_{u}}\left(\xi_{1} f_{u}^{\mathrm{LoS}}+\xi_{2} f_{u}^{\mathrm{NLoS}}\right) d_{u}^{-\alpha / 2} \tilde{h}_{u} x_{u}+n_{u},
$$

where $P_{u}$ denotes the transmit power of drone $u$, while $x_{u}$ represents its transmitted data. Moreover, $n_{u} \sim \mathcal{N}\left(0, N_{0}\right)$ is the additive white Gaussian noise (AWGN). Then, the signalto-noise ratio (SNR) is given by:

$$
\mathrm{SNR}_{u}=\frac{P_{u}\left(\xi_{1} f_{u}^{L o S}+\xi_{2} f_{u}^{N L o S}\right)^{2} d_{u}^{-\alpha}\left|\tilde{h}_{u}\right|^{2}}{N_{0}} .
$$

Let us now define the outage event.

Proposition 1. The transmission between the gateway $u$ and the AP fails, when the receiver's $\mathrm{SNR}_{u}$ is below a given threshold $\beta$, which is defined as an outage event. This event occurs with the probability $f_{u}$, which can be expressed as:

$$
f_{u}=1-Q\left(\frac{A}{\sigma}, \frac{N_{0} \beta d^{\alpha}}{P_{u}\left(\xi_{1} f_{u}^{L o S}+\xi_{2} f_{u}^{N L o S}\right)^{2}}\right),
$$

where

$$
Q(u, v)=\int_{v}^{\infty} x \exp \left[-\frac{1}{2}\left(x^{2}+u^{2}\right)\right] I_{0}(x u) d x
$$


is the Marcum $Q$ function.

Proof: See Appendix A.

Proposition 1 indicates that the data transmission between the gateway UAV and the AP may fail because of the stochastic character of the channel. Hence, an effective retransmission scheme is needed for improving the reliability of the communication [40]. The automatic repeat request (ARQ) scheme allows the gateway UAV to retransmit the packet either until it is successfully received or a maximum number of retransmissions $N$ is reached. The number of ARQ rounds required depends on the channel conditions.

\section{B. Required Transmission Time and Energy Consumption}

The energy consumption and the time required for data transmission are the main parameters in selecting the gateway. Given that the energy consumption and the time required for transmitting a single packet depends on the number of ARQ attempts, only the average performance is considered in the following. We first calculate the average number $\bar{N}_{u}^{\text {trans }}$ of packet retransmissions of the gateway $u$, which can be expressed as:

$$
\begin{aligned}
\bar{N}_{u}^{\text {trans }}= & 1 \cdot \operatorname{Pr}\left(S^{1}\right)+2 \cdot \operatorname{Pr}\left(F^{1}, S^{2}\right)+\cdots \\
& +(N-1) \cdot \operatorname{Pr}\left(F^{1}, F^{2}, \ldots, S^{N-1}\right) \\
& +N \cdot\left[\operatorname{Pr}\left(F^{1}, F^{2}, \ldots, S^{N}\right)+\operatorname{Pr}\left(F^{1}, F^{2}, \ldots, F^{N}\right)\right] \\
= & 1 \cdot \operatorname{Pr}\left(S^{1}\right)+2 \cdot \operatorname{Pr}\left(F^{1}, S^{2}\right)+\cdots \\
& +(N-1) \cdot \operatorname{Pr}\left(F^{1}, F^{2}, \ldots, S^{N-1}\right) \\
& +N \cdot \operatorname{Pr}\left(F^{1}, F^{2}, \ldots, F^{N-1}\right),
\end{aligned}
$$

where $\operatorname{Pr}\left(F^{1}, F^{2}, \ldots, F^{m-1}, S^{m}\right)$ denotes the probability that the transmission fails at the 1 st $, \ldots,(m-1)$-th rounds but succeeds at the $m$-th round. Since the channel state information (CSI) at each ARQ round is i.i.d., the failure and success probability of each time transmission are independent of each other, i.e. $\operatorname{Pr}\left(F^{1}, F^{2}, \ldots, F^{m-1}, S^{m}\right)=$ $\operatorname{Pr}\left(F^{1}\right) \operatorname{Pr}\left(F^{2}\right) \cdots \operatorname{Pr}\left(F^{m-1}\right) \operatorname{Pr}\left(S^{m}\right)=f_{u}^{m-1}\left(1-f_{u}\right)=$ $f_{u}^{m-1}-f_{u}^{m}$. Therefore, $\bar{N}_{\text {trans }}$ can be further expressed as:

$$
\begin{aligned}
\bar{N}_{u}^{\text {trans }}= & \left(1-f_{u}\right)+2\left(f_{u}-f_{u}^{2}\right)+\cdots \\
& +(N-1)\left(f_{u}^{N-2}-f_{u}^{N-1}\right)+N f_{u}^{N-1} \\
= & 1+f_{u}+\cdots+f_{u}^{N-1}=\frac{1-f_{u}^{N}}{1-f_{u}} .
\end{aligned}
$$

Let $T_{u}^{\text {one }}$ be the average time required for transmitting a single packet, which is given by:

$$
T_{u}^{\mathrm{one}}=\bar{N}_{u}^{\text {trans }} \cdot T^{\text {travel }},
$$

where $T^{\text {travel }}$ denotes the 'flight-time' of a packet. Assuming that a packet contains $K$ bits, $T^{\text {travel }}$ can be expressed as:

$$
T^{\text {travel }}=\frac{K}{B \log _{2}(1+\beta)} .
$$

If $A_{i}(t)$ packets of a FANET have to be transmitted, the total transmission time is given by:

$$
\begin{aligned}
T_{u}^{\text {total }} & =A_{i}(t) \cdot T_{u}^{\text {one }} \\
& =A_{i}(t) \cdot \frac{1-f_{u}^{N}}{1-f_{u}} \cdot \frac{K}{B \log _{2}(1+\beta)} .
\end{aligned}
$$

The energy consumption of the gateway UAV $u$ includes both the hovering energy and the transmission energy, but naturally the hovering energy dominates the total energy consumption, which depends on the UAV type. We assume that the hovering power of the gateway UAV $u$ is $P_{u}^{\text {hover }}$, and hence the hovering energy consumption in each time slot can be calculated by:

$$
\mathcal{E}_{u}^{\text {hover }}=P_{u}^{\text {hover }} \Delta t
$$

By contrast, the energy consumption of transmitting $A_{i}(t)$ packets is calculated by:

$$
\begin{aligned}
\mathcal{E}_{u}^{\text {trans }} & =A_{i}(t) \cdot P_{u} \cdot T_{u}^{\text {one }} \\
& =A_{i}(t) \cdot P_{u} \cdot \frac{1-f_{u}^{N}}{1-f_{u}} \cdot \frac{K}{B \log _{2}(1+\beta)} .
\end{aligned}
$$

Therefore, the total energy consumption in each time slot can be expressed as:

$$
\begin{aligned}
\mathcal{E}_{u}^{\text {total }} & =\mathcal{E}_{u}^{\text {hover }}+\mathcal{E}_{u}^{\text {trans }} \\
& =P_{u}^{\text {hover }} \Delta t+A_{i}(t) \cdot P_{u} \cdot \frac{1-f_{u}^{N}}{1-f_{u}} \cdot \frac{K}{B \log _{2}(1+\beta)} .
\end{aligned}
$$

\section{An Energy-Efficient Gateway Selection Scheme}

In this section, we design an energy-efficient gateway selection scheme. Our objective is to minimize the total energy consumption under specific energy and transmission time constraints. Let $\mathcal{E}_{u}^{\text {Battery }}$ denote the residual battery energy of $u$, which should have sufficient energy both for hovering and data transmission, i.e. $\mathcal{E}_{u}^{\text {Battery }}>\mathcal{E}_{u}^{\text {total }}$. Moreover, the residual energy of the selected gateway $u$ should exceed a threshold $\mathcal{E}^{\text {th }}$ for its operation in the next time slot. Additionally, the time required for transmission should not exceed a pre-defined threshold $T^{\text {th }}$. Let $\varsigma_{u}$ be a Boolean decision variable, which is defined as follows:

$$
\varsigma_{u}= \begin{cases}1 & \text { If } u \text { is selected as the gateway, } \\ 0 & \text { Otherwise. }\end{cases}
$$

Then the gateway selection can be formulated as a linear integer problem given by:

$$
\begin{aligned}
\mathcal{P} 1: \quad \min _{\varsigma_{u}} & \sum_{u \in \mathcal{G}_{i}} \mathcal{E}_{u}^{\text {total }} \cdot \varsigma_{u} \\
\text { s.t. } \quad & \sum_{u \in \mathcal{G}_{i}}\left(\mathcal{E}_{u}^{\text {Battery }}-\mathcal{E}_{u}^{\text {total }}\right) \cdot \varsigma_{u} \geq \mathcal{E}^{\text {th }}, \\
& T_{u}^{\text {total }} \leq T^{\text {th }}, \quad \forall u \in \mathcal{G}_{i}, \\
& \sum_{u \in \mathcal{G}_{i}} \varsigma_{u}=1, \\
& \varsigma_{u} \in\{0,1\}, \quad \forall u \in \mathcal{G}_{i} .
\end{aligned}
$$


Specifically, Eq. (22a) ensures that the selected gateway has sufficient residual energy for continuing its operation, while Eq. (22b) guarantees that the transmission latency is lower than the threshold. Eq. (22c) requires that only one UAV can be selected as the gateway UAV in a FANET, and Eq. (22d) indicates that each UAV only has a binary selection space.

Again, problem $\mathcal{P} \mathbf{1}$ is an integer programming problem, which can be solved by brute-force search. In fact, since the set $\mathcal{G}_{i}$ is given, the size of the search space is $\left|\mathcal{G}_{i}\right|$, where $|\cdot|$ is the cardinality of a set. Therefore, the exhaustive search will have a complexity order of $\mathcal{O}(1)$, which is suitable for the computationally-limited UAV network. Moreover, $\mathcal{P} 1$ can also be converted to a convex optimization problem. Since only a single of $\varsigma_{u}$ has the value of one and the other $\varsigma_{u}$ have a value of zero, we can relax the integer constraint of Eq. (22d) to a continuous convex constraint $0 \leq \varsigma_{u} \leq 1$ without affecting the optimal outcome or increasing computational complexity. Hence, the optimal gateway can also be found by Algorithm 1.

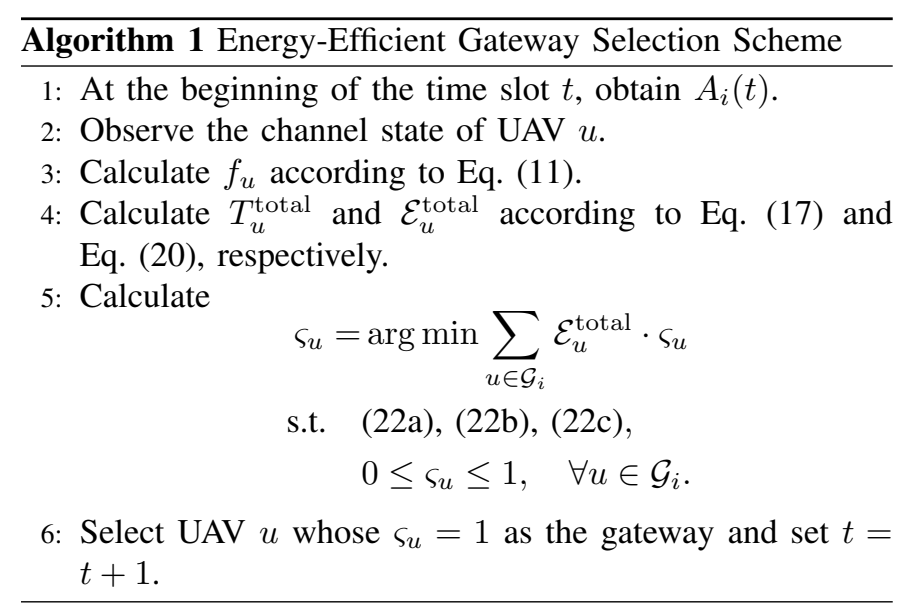

\section{TAsk Scheduling and Resource Allocation SCHEME}

As mentioned before, task scheduling is an important issue in heterogeneous cloud aided systems. Specifically, if all the packets are locally processed in the edge cloud, it is difficult to maintain the system's stability because both the computational capability and the buffer capacity of the edge cloud are limited. On the other hand, if all the packets are offloaded and tackled by the remote cloud, the power consumption may become excessive. In this section, our goal is to find an optimal scheme to determine the percentage of packets processed in the edge cloud as well as the amount of computational resources allocated for different tasks considering both the average power consumption and the cloud execution delay.

\section{A. Average Power Consumption and Cloud Execution Delay}

The power consumption of processing the packets arriving from FANET $i$ in time slot $t$ is composed of two parts, i.e. the power consumption in the edge cloud and the power consumption in the remote cloud. Let $P_{i}(t)=P_{e, i}(t)+P_{r, i}(t)$ denote the total power consumption of fulfilling the task requested by UAV $i$ in time slot $t$. Considering a total number of $I$ FANETs, the system's power consumption can be calculated as $P(t)=\sum_{i=1}^{I} P_{i}(t)$. Then, the time-averaged power consumption is given by:

$$
\bar{P}=\lim _{t \rightarrow \infty} \frac{1}{t} E\left[\sum_{\tau=0}^{t-1} P(t)\right] .
$$

Since the Internet's transmission delay in the remote cloud is assumed to be constant, the average execution delay essentially hinges on the sojourn time in each edge cloud's queue. Relying on Little's Law [38], we use the time-averaged queue length in the task buffer as a metric of the average execution delay, which is expressed as:

$$
\bar{Q}_{i}=\lim _{t \rightarrow \infty} \frac{1}{t} E\left[\sum_{\tau=0}^{t-1} Q_{i}(t)\right] .
$$

Definition 1. The queueing system of FANET $i$ is strongly stable in the edge cloud, if [36]:

$$
\lim _{t \rightarrow \infty} \frac{1}{t} E\left[\sum_{\tau=0}^{t-1} Q_{i}(t)\right]<\infty .
$$

Note that if all the queues are stable, the system's average service rate is equal to the packet arrival rate, yielding:

$$
\lim _{t \rightarrow \infty} \frac{1}{t} \sum_{\tau=0}^{t-1} u_{i}(t)=\lim _{t \rightarrow \infty} \frac{1}{t} \sum_{\tau=0}^{t-1} A_{e, i}(t),
$$

which guarantees that the arriving packets can be processed within a finite time delay.

In this paper, we aim for finding a task scheduling and resource allocation scheme for the sake of minimizing the time-averaged power consumption under the constraint that all the tasks can be executed within a finite time delay. Let $\boldsymbol{\eta}(t)=\left[\eta_{1}(t), \eta_{2}(t), \ldots, \eta_{I}(t)\right]$, where $\eta_{i}(t)=\frac{A_{e, i}(t)}{A_{i}(t)}$ denotes the ratio of packets processed in the edge cloud, and $\boldsymbol{n}(t)=\left[n_{1}(t), n_{2}(t), \ldots, n_{I}(t)\right]$. Hence, this scheduling and resource allocation problem can be formulated as:

$$
\begin{array}{ll}
\mathcal{P} 2: & \min _{\boldsymbol{\eta}(t), \boldsymbol{n}(t)} \bar{P} \\
\text { s.t. } & 0 \leq \eta_{i}(t) \leq 1, \quad i=1,2, \ldots, I, t \in \mathcal{T}, \\
& \sum_{i=1}^{I} n_{i}(t) \leq n_{\max }, \quad t \in \mathcal{T}, \\
& \lim _{t \rightarrow \infty} \frac{1}{t} E\left[\sum_{\tau=0}^{t-1} Q_{i}(t)\right]<\infty, \quad i=1,2, \ldots, I .
\end{array}
$$

To elaborate, (27a) requires that the packets arriving from FANET $i$ should be processed either by the edge cloud or by the remote cloud, while (27b) indicates that the sum of the number of CPU-cycles does not exceed the total computational capacity. Moreover, (27c) indicates that the packets can be processed in the edge cloud within a finite delay.

\section{B. Task Scheduling and Resource Allocation Scheme Based on Lyapunov Optimization}

Problem $\mathcal{P} \mathbf{2}$ is a stochastic optimization problem since both $\boldsymbol{\eta}(t)$ and $\boldsymbol{n}(t)$ are time-varying, and hence the optimal 
strategy is temporally corrected. In this section, we design an online algorithm to determine the optimal task scheduling and resource allocation strategy of each time slot based on Lyapunov optimization. To elaborate, Lyapunov optimization is designed for the greedy minimization of the queue backlog in each time slot by solving a deterministic problem. The detailed discussions and theorems of Lyapunov optimization can be found in [36].

First of all, we define the function $L[\boldsymbol{Q}(t)]$ as the sum of the squared number of packets in the queue:

$$
L[\boldsymbol{Q}(t)]=\frac{1}{2} \sum_{i=0}^{I} Q_{i}^{2}(t) .
$$

Then, the conditional Lyapunov drift in time slot $t$ can be expressed by:

$$
\Delta[\boldsymbol{Q}(t)]=E[L\{\boldsymbol{Q}(t+1)\}-L\{\boldsymbol{Q}(t)\} \mid \boldsymbol{Q}(t)] .
$$

If we minimize $\Delta[\boldsymbol{Q}(t)]$ in each time slot, this may stabilize the system. However, the average power consumption might be unnecessarily high. Alternatively, we may define a drift-pluspenalty function $\Delta_{V}[\boldsymbol{Q}(t)]$ to strike a tradeoff between the power-cost and queue backlog. The drift-plus-penalty function may be defined as:

$$
\Delta_{V}[\boldsymbol{Q}(t)]=\Delta[\boldsymbol{Q}(t)]+V \cdot E[P(t) \mid \boldsymbol{Q}(t)],
$$

where $V$ is the control weighting parameter that represents how much we emphasize the power consumption. A large value of $V$ is beneficial of optimizing the average power consumption at the expense of high average delay. Thus, the drift-plus-penalty function provably strikes a balance between the power consumption and execution delay in the cloud.

Lemma 1. For any $\boldsymbol{\eta}(t)$ and $\boldsymbol{n}(t)$ satisfying $\eta_{i}(t) \in$ $[0,1], n_{i}(t) \in\left[0, n_{\max }\right], i=1,2, \ldots, I, \Delta_{V}[\boldsymbol{Q}(t)]$ is upper bounded by:

$$
\begin{aligned}
& \Delta_{V}[\boldsymbol{Q}(t)] \leq \\
& \frac{1}{2} E\left[\sum_{i=1}^{I}\left(A_{e, i}^{2}(t)+\mu_{i}^{2}(t)-2 A_{e, i}(t) \mu_{i}(t)\right) \mid \boldsymbol{Q}(t)\right] \\
& \quad-E\left[\sum_{i=1}^{I} Q_{i}(t)\left(\mu_{i}(t)-A_{e, i}(t)\right) \mid \boldsymbol{Q}(t)\right] \\
& \quad+V \cdot E[P(t) \mid \boldsymbol{Q}(t)] .
\end{aligned}
$$

Proof: See Appendix B.

For the sake of achieving a superior task scheduling and resource allocation, we conceived Algorithm 2 aiming for the greedy minimization of the upper bound of $\Delta_{V}[\boldsymbol{Q}(t)]$ in each time slot, where we have:

$$
\begin{aligned}
h_{c}[\boldsymbol{\eta}(t), \boldsymbol{n}(t)] & =\frac{1}{2} \sum_{i=1}^{I}\left[A_{e, i}^{2}(t)+\mu_{i}^{2}(t)-2 A_{e, i}(t) \mu_{i}(t)\right] \\
& -\sum_{i=1}^{I}\left[Q_{i}(t)\left(\mu_{i}(t)-A_{e, i}(t)\right)\right]+V \cdot P(t) .
\end{aligned}
$$

Note that in Algorithm 2, solving the stochastic optimization problem $\mathcal{P} 2$ is reduced to deal with a deterministic optimiza- tion problem $\mathcal{P} 3$ in each time slot. Since $\mathcal{P} 3$ is a convex optimization problem, Algorithm 2 is able to find the optimal solution in each time slot with a complexity order of $\mathcal{O}\left(I^{2}\right)$. By appropriately adjusting the value of $V$, we can strike a power-vs-delay trade-off. Moreover, the proposed algorithm is capable of minimizing the power consumption and of driving the queue backlog towards a low buffer content corresponding to a low delay.

$\begin{aligned} & \text { Algorithm } 2 \text { Task Scheduling and Resource Allocation } \\ & \text { Scheme }\end{aligned}$
$\begin{aligned} \text { 1: At the beginning of the time slot } t, \text { obtain }\left\{Q_{i}(t)\right\} \text { a } \\ \left\{A_{i}(t)\right\} . \\ \text { 2: Calculate } \\ \mathcal{P} 3: \quad\{\boldsymbol{\eta}(t), \boldsymbol{n}(t)\}=\arg \min \quad h_{c}[\boldsymbol{\eta}(t), \boldsymbol{n}(t)] \\ \text { s.t. } \quad(27 \mathrm{a}),(27 \mathrm{~b}) .\end{aligned}$

3: Update $\left\{Q_{i}(t)\right\}$ according to Eq. (7) and set $t=t+1$.

\section{A Low-Complexity Iterative Algorithm}

Problem $\mathcal{P} 3$ is a convex optimization problem and can be solved by interior-point methods [41]. However, the generic convex-problem solution algorithms suffer from high computational cost. In this section, we conceive a low-complexity algorithm for solving $\mathcal{P} 3$ based on the structured nature of the problem. Since $\mathcal{P} \mathbf{2}$ is a joint task-scheduling and resource allocation problem, its minimization can be achieved by searching through the solution-space by exploiting the decreasing gradient direction of either of them. Hence, by fixing one of the two variables, we can obtain the optimal value of the other one.

Substituting Eq. (5), Eq. (6) and Eq. (8) into Eq. (32), we have:

$$
\begin{aligned}
& h_{c}[\boldsymbol{\eta}(t), \boldsymbol{n}(t)]= \\
& \frac{1}{2} \sum_{i=1}^{I}\left[\eta_{i}^{2}(t) A_{i}^{2}(t)+\Delta t^{2} n_{i}^{2}(t) L_{i}^{-2}-2 \eta_{i}(t) A_{i}(t) \Delta t n_{i}(t) L_{i}^{-1}\right] \\
& -\sum_{i=1}^{I}\left[Q_{i}(t)\left(\Delta t n_{i}(t) L_{i}^{-1}-\eta_{i}(t) A_{i}(t)\right)\right] \\
& +V \sum_{i=1}^{I}\left[\kappa n_{i}^{3}(t)+C e^{\left(1-\eta_{i}(t)\right) A_{i}(t)}\right] .
\end{aligned}
$$

Optimal resource allocation : By fixing $\boldsymbol{\eta}(t)$, the optimization goal of $\mathcal{P} \mathbf{3}$ reduces to:

$$
\begin{aligned}
& h_{c}[\boldsymbol{\eta}(t), \boldsymbol{n}(t) \mid \boldsymbol{\eta}(t)]= \\
& \frac{1}{2} \sum_{i=1}^{I}\left[\Delta t^{2} n_{i}^{2}(t) L_{i}^{-2}-2 \eta_{i}(t) A_{i}(t) \Delta t n_{i}(t) L_{i}^{-1}\right] \\
& -\sum_{i=1}^{I} Q_{i}(t) \Delta t n_{i}(t) L_{i}^{-1}+V \sum_{i=1}^{I} \kappa n_{i}^{3}(t) .
\end{aligned}
$$

Hence, the resultant single-variable optimization problem can 
be formulated as:

$$
\begin{gathered}
\mathcal{P} 4: \min _{\boldsymbol{n}(t)} h_{c}[\boldsymbol{\eta}(t), \boldsymbol{n}(t) \mid \boldsymbol{\eta}(t)] \\
\text { s.t. } \sum_{i=1}^{I} n_{i}(t) \leq n_{\max } .
\end{gathered}
$$

Problem $\mathcal{P} 4$ can be solved using the Lagrangian dual method. The Lagrangian function of $\mathcal{P} \mathbf{4}$ can be written as:

$$
L[\boldsymbol{n}(t), \lambda]=h_{c}[\boldsymbol{\eta}(t), \boldsymbol{n}(t) \mid \boldsymbol{\eta}(t)]+\lambda\left(\sum_{i=1}^{I} n_{i}(t)-n_{\max }\right),
$$

where $\lambda$ is the Lagrangian multiplier associated with the constraint Eq. (35a). Since $\mathcal{P} 4$ is a convex optimization problem, the optimal solution of the original and of the dual problem, i.e. of $\hat{n}_{i}(t)$ and $\hat{\lambda}$, should satisfy the Karush-KuhnTucker (KKT) conditions:

$$
\left\{\begin{aligned}
\hat{\lambda} & \geq 0 \\
\sum_{i=1}^{I} \hat{n}_{i}(t)-n_{\max } & \leq 0 \\
\hat{\lambda}\left(\sum_{i=1}^{I} \hat{n}_{i}(t)-n_{\max }\right) & =0 \\
\left.\frac{\partial h_{c}(\boldsymbol{\eta}(t), \boldsymbol{n}(t) \mid \boldsymbol{\eta}(t))}{\partial n_{i}(t)}\right|_{n_{i}(t)=\hat{n}_{i}(t)}+\hat{\lambda} & =0
\end{aligned}\right.
$$

Substituting Eq. (34) into Eq. (37), $\hat{n}_{i}(t)$ can be expressed as Eq. (38). Hence, we only have to determine $\hat{\lambda}$. In Eq. (37), if $\lambda=0$ and $\sum_{i=1}^{I} n_{i}(t)-n_{\max } \leq 0, \hat{\lambda}=0$ is the optimal solution. Otherwise, $\hat{\lambda}$ can be determined with the aid of the bisection search approach. The region of the search approach spans $\left[\lambda_{L}, \lambda_{U}\right]$, where $\lambda_{L}=0$ and $\lambda_{U}$ satisfies $\sum_{i=1}^{I} n_{i}(t)-$ $n_{\max } \leq 0$. Thus, the available selection of $\lambda_{U}$ is given by:

$$
\lambda_{U}=\max _{i}\left\{\Delta t L_{i}^{-1}\left(\eta_{i}(t) A_{i}(t)+Q_{i}(t)\right)\right\} .
$$

The search for the optimal $\hat{\lambda}$ is terminated if:

$$
\frac{\left|\sum_{i=1}^{I} n_{i}(t)-n_{\max }\right|}{n_{\max }} \leq \delta,
$$

where $\delta$ is the search precision.

Optimal Task Scheduling: By fixing $\boldsymbol{n}(t)$, the optimization

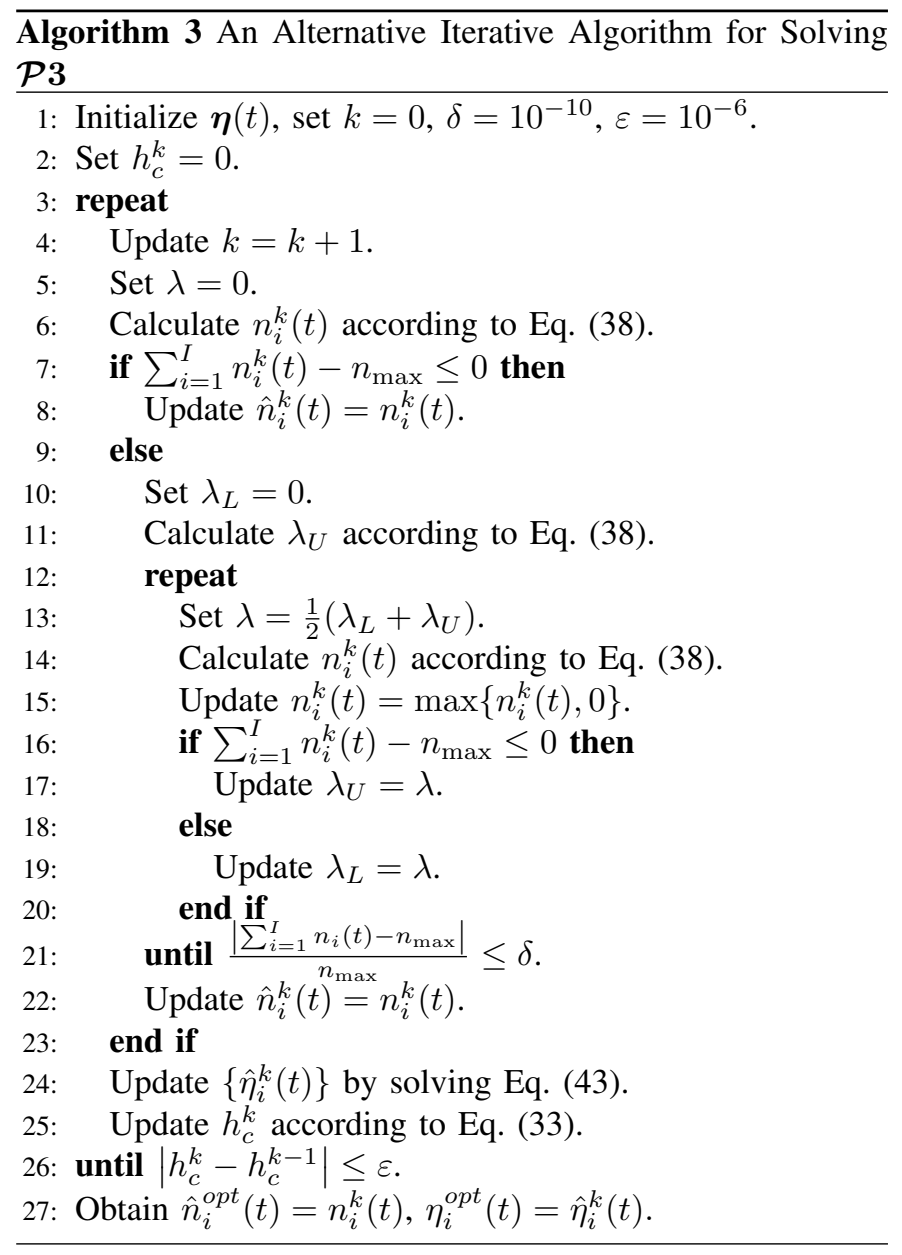

objective reduces to:

$$
\begin{aligned}
& h_{c}[\boldsymbol{\eta}(t), \boldsymbol{n}(t) \mid \boldsymbol{n}(t)]= \\
& \frac{1}{2} \sum_{i=1}^{I}\left[\eta_{i}^{2}(t) A_{i}^{2}(t)-2 \eta_{i}(t) A_{i}(t) \Delta t n_{i}(t) L_{i}^{-1}\right] \\
& +\sum_{i=1}^{I}\left[Q_{i}(t) \eta_{i}(t) A_{i}(t)\right]+C V \sum_{i=1}^{M} e^{\left(1-\eta_{i}(t)\right) A_{i}(t)} .
\end{aligned}
$$

Then, the optimization problem can be rewritten as:

$$
\begin{aligned}
\mathcal{P} 5: & \min _{\boldsymbol{\eta}(t)} h_{c}[\boldsymbol{\eta}(t), \boldsymbol{n}(t) \mid \boldsymbol{n}(t)] \\
\text { s.t. } & 0 \leq \eta_{i}(t) \leq 1, \quad i=1,2, \ldots, I .
\end{aligned}
$$

Since $\eta_{i}(t)^{\prime} s$ are i.i.d., the optimal solutions can be achieved

$$
\hat{n}_{i}(t)=\frac{-\Delta t^{2} L_{i}^{-2}+\sqrt{\Delta t^{4} L_{i}^{-4}+12 V \kappa\left[\Delta t L_{i}^{-1}\left(\eta_{i}(t) A_{i}(t)+Q_{i}(t)\right)-\hat{\lambda}\right]}}{6 V \kappa} .
$$




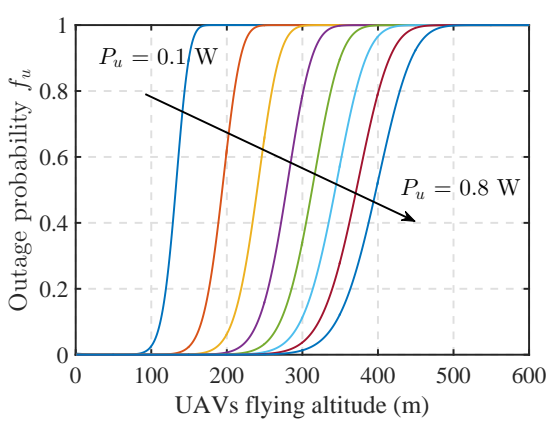

(a) $\mathrm{R}=50 \mathrm{~m}$

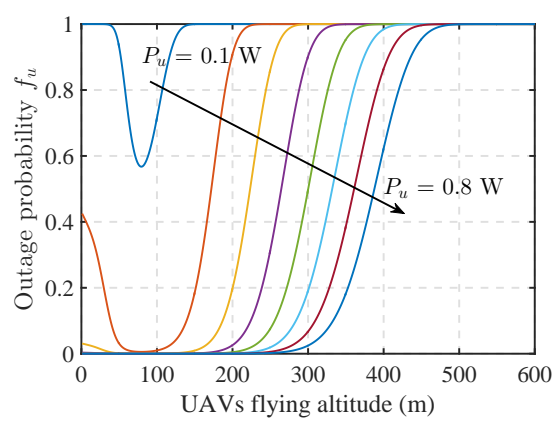

(b) $\mathrm{R}=100 \mathrm{~m}$

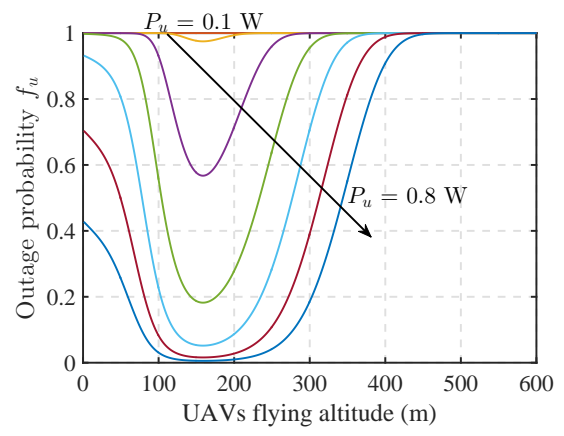

(c) $\mathrm{R}=200 \mathrm{~m}$

Fig. 2. Outage probability (calculated by Eq. (11)) versus UAV's hovering altitude parameterized by the UAV's transmit power, where (a), (b) and (c) represents the cases that the horizontal distance between the UAV and the AP is $50 \mathrm{~m}, 100 \mathrm{~m}$ and $200 \mathrm{~m}$, respectively.

by solving:

$$
\begin{aligned}
& \frac{\partial h_{c}[\boldsymbol{\eta}(t), \boldsymbol{n}(t) \mid \boldsymbol{n}(t)]}{\partial \eta_{i}(t)}= \\
& \quad A_{i}(t) C V e^{\left[1-\eta_{i}(t)\right] A_{i}(t)}-\eta_{i}(t) A_{i}^{2}(t)+A_{i}(t) \Delta t n_{i}(t) L_{i}^{-1} \\
& \quad-Q_{i}(t) A_{i}(t)=0 .
\end{aligned}
$$

Eq. (43) is a transcendental equation, which can be readily solved by numerical methods such as Newton's method or Secant method. The detailed procedure is omitted in this paper. Let $\eta_{i}^{*}(t)$ be the solution of Eq. (43), for which we have $\left.\frac{\partial^{2} h_{c}[\boldsymbol{\eta}(t), \boldsymbol{n}(t) \mid \boldsymbol{n}(t)]}{\partial \eta_{i}^{2}(t)}\right|_{\eta_{i}(t)=\eta_{i}^{*}(t)} \geq 0$. Hence, $\eta_{i}^{*}(t)$ is the global minimum point. Considering $\eta_{i}(t) \in[0,1]$, the optimal solution is given by:

$$
\hat{\eta}_{i}(t)=\max \left\{0, \min \left\{\eta_{i}^{*}(t), 1\right\}\right\}, i=1,2, \ldots, I .
$$

Note that although problem $\mathcal{P} 4$ and $\mathcal{P} 5$ are solved optimally, the solutions obtained in Eq. (38) and Eq. (44) may not be the optimal ones to $\mathcal{P} \mathbf{3}$ for the existence of the coupling term. Therefore, we provide an iterative algorithm for finding the optimal solutions for $\mathcal{P} 3$. Our solution is detailed in Algorithm 3. Since both the subproblems, e.g., problem $\mathcal{P} 4$ and $\mathcal{P} 5$, are solved optimally, Algorithm 3 is guaranteed to converge to the optimal solutions to problem $\mathcal{P} 3$ with a complexity order of $\mathcal{O}(I L)$, where $L$ is the number of iterations, which is usually far less than $I$.

\section{Simulation Results}

In this section, we provide numerical results for characterizing the proposed algorithms. We assume that all the UAVs in $I$ FANETs are randomly distributed in a circular area with a radius of $R$ and hover at a height of $\mathrm{H}$. We assume urban environment with $\phi=11.95$ and $\varphi=0.14$ [5]. The path loss coefficients are $\xi_{1}=7 \times 10^{-5}, \xi_{2}=3.5 \times 10^{-5}$ and the path loss exponent is $\alpha=2$. The mean and variance of the Gaussian random signals are set to be $\mu_{1}=\mu_{2}=8$ and $\sigma^{2}=5$, respectively. Hence the k-factor of the Rician fading channel is $\frac{\mu_{1}^{2}+\mu_{2}^{2}}{2 \sigma^{2}}=12.8$. The power of AWGN is $N_{0}=B n_{0}$, where $n_{0}=-174 \mathrm{dBm} / \mathrm{Hz}$ is the AWGN's power spectral density and $B=10 \mathrm{MHz}$ is the bandwidth allocated to each FANET. The SNR threshold is set to $\beta=5$ $\mathrm{dB}$. For each FANET, the length of one time slot is $30 \mathrm{~ms}$,

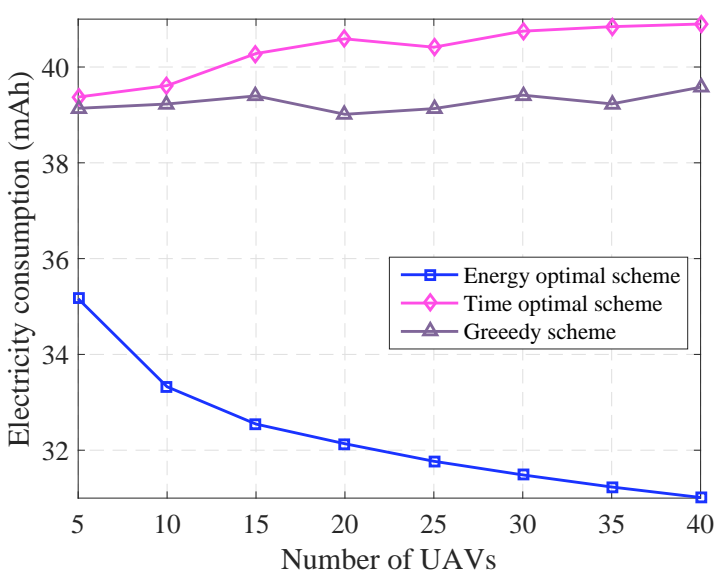

Fig. 3. Energy consumption (calculated by Eq. (20)) of different gateway selection schemes versus the number of UAVs.

and we assume that $A_{i, \min }=10, A_{i, \max }=100$. Moreover, $A_{i}(t)$ is uniformly distributed within the range of $[10,100]$. The number of bits that a packet contains is $K=5000$ and the maximum number of retransmission rounds of the ARQ scheme is $N=8$. Additionally, let $\kappa=10^{-27}, L=3 \times 10^{6}$, $n_{\max }=10^{10}$ and $C=10^{-20}$.

\section{A. Performance of Gateway Selection Scheme}

Since the transmission energy and transmission time are related to the link quality, e.g. the outage probability, which further depends on the UAV's transmit power and hovering altitude, we first explore the interaction between them. The corresponding results are shown in Fig. 2. It is observed from Fig. 2 that as expected, the outage probability decreases with the transmit power. Moreover, it is also observed that the outage probability first decreases and then increases with the hovering altitude. This is plausible because when the hovering altitude increases from a low height, the probability of LoS links is increased. However, when the hovering altitude exceeds a certain height, the path loss increase outweights the gains brought about by having more LoS links. This phenomenon indicates that the hovering altitudes of UAVs has to be carefully designed for maximizing the channel gain.

We then evaluate the performance of the gateway selection 


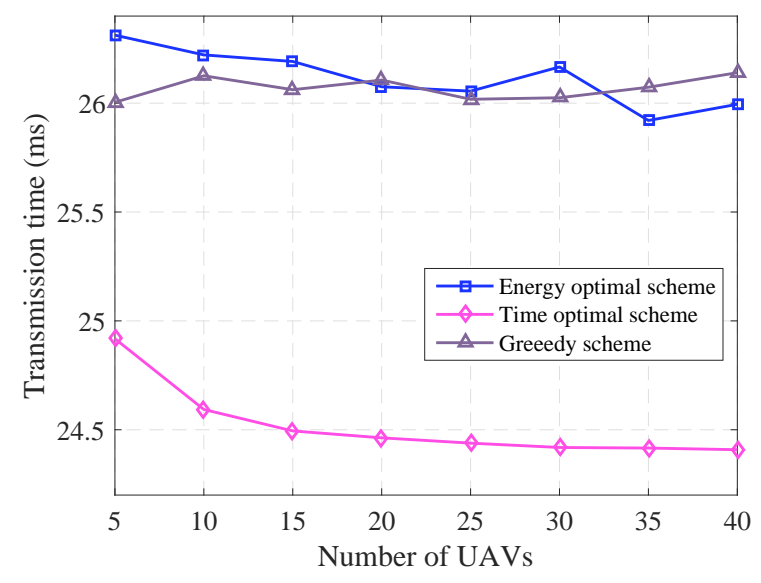

Fig. 4. Required transmission time (calculated by Eq. (17)) of different gateway selection schemes versus the number of UAVs.

scheme described in Algorithm 1. Without loss of generality, we highlight the performance of a single FANET in one time slot. We assume that the horizontal distances between the AP and UAVs are randomly distributed between $[150,200] \mathrm{m}$ and the hovering altitudes are randomly distributed between $[200,300] \mathrm{m}$. The energy of the UAVs' batteries are randomly distributed within $[5000,8000] \mathrm{mAh}$ with an output voltage of $15 \mathrm{~V}$. The energy threshold and time thresholds are $4500 \mathrm{mAh}$ and $30 \mathrm{~ms}$, respectively. The hovering power of the UAV is $40 \mathrm{~W}$, while the transmit power is randomly distributed within $[0.5,0.8] \mathrm{W}$. The results are averaged over 500 simulation$\mathrm{s}$. The required transmission energy ${ }^{1}$ and transmission time performances are characterized in Fig. 3 and Fig. 4. The performances of our proposed energy-optimal gateway selection scheme and of other schemes, i.e. of the time-optimal scheme and of the greedy scheme, are also compared at the same time. Specifically, the time-optimal scheme selects the specific UAV that needs the lowest transmission time as the gateway, while the greedy scheme randomly chooses a UAV that satisfies the energy and transmission time constraints considered. It is shown in Fig. 3 and Fig. 4 that our proposed energy-optimal scheme consumes the least energy, while the time-optimal scheme requires the least transmission time. Moreover, the performance of the greedy scheme falls somewhere in between. When jointly considering both the energy consumption and transmission time, our proposed energy-optimal scheme consumes nearly $10 \mathrm{mAh}$ lower energy than that of the other two schemes at the cost of less than 2 ms higher transmission time than that of the time-optimal scheme. This is beneficial in the context of a battery-constrained environments. Therefore, our proposed scheme is superior to other two schemes.

\section{B. Performance of Task Scheduling and Resource Allocation scheme}

This section analyzes the performance of the proposed task scheduling and resource allocation scheme described in

\footnotetext{
${ }^{1}$ The energy consumption is represented by the electricity consumption in our simulations, they can be mutually transformed since the output voltage and the length of one time slot are known as given parameters.
}

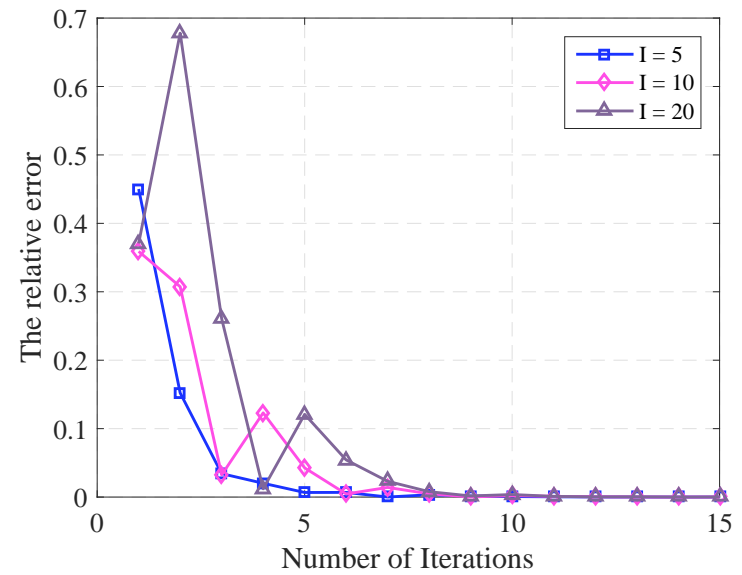

Fig. 5. The convergence of the bisection search process in Algorithm 3 parameterized by different number of FANETs.

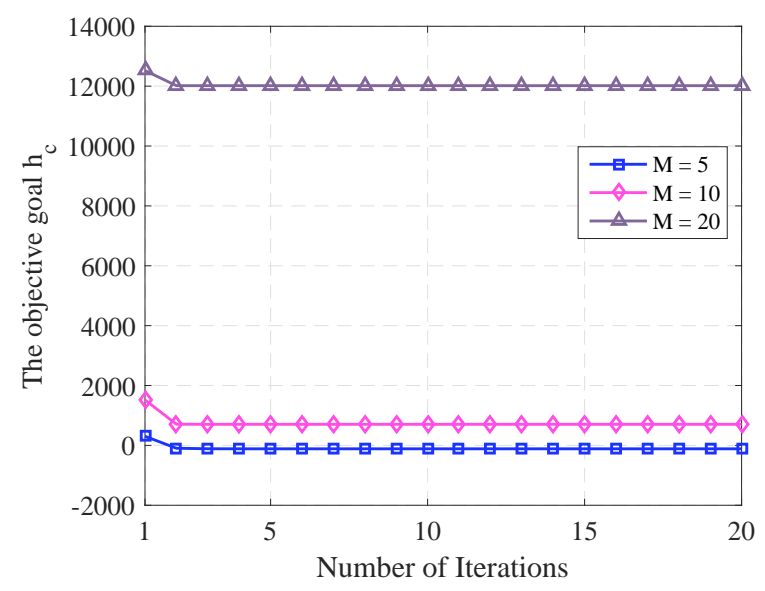

Fig. 6. The convergence of Algorithm 3 parameterized by different number of FANETs.

Algorithm 2 and Algorithm 3. In Fig. 5, we first characterize the convergence of the bisection search process described in the inner loop of Algorithm 3. The convergence of the outer loop of Algorithm 3 is shown in Fig. 6, where we set the control parameter of the drift-plus-penalty function in Eq. (30) as $V=500$. Fig. 5 shows that the bisection based search finds the optimal solutions of $\mathcal{P} 4$ within 15 loops at a relative error below $10^{-10}$. It is much faster than solving $\mathcal{P} 4$ using other optimization algorithm considering the huge region of search. Additionally, it can be inferred from Fig. 6 that given a set of randomly generated $\boldsymbol{\eta}^{0}(t)$, Algorithm 3 is capable of achieving convergence in as few as 2 steps. Moreover, increasing the number of drones in the FANETs has almost no influence on the rate of convergence. Therefore, our algorithm achieves fast convergence at a lower computational cost compared to solving $\mathcal{P} 3$ using interior-point methods.

Fig. 7 shows the time-averaged power consumption of task execution and the average queue length per user versus the control parameter, where we set $I=10$. The results are averaged over 100 time slots. It can be observed that the timeaveraged power consumption decreases upon increasing $V$, 
(a)
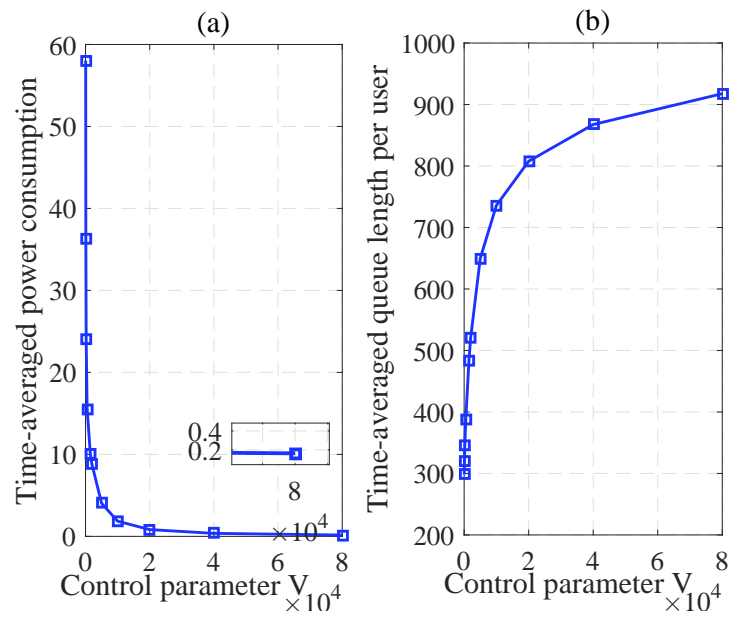

Fig. 7. Time-averaged power consumption (calculated by Eq. (23)) and queue length (calculated by Eq. (24)) versus the control parameter.

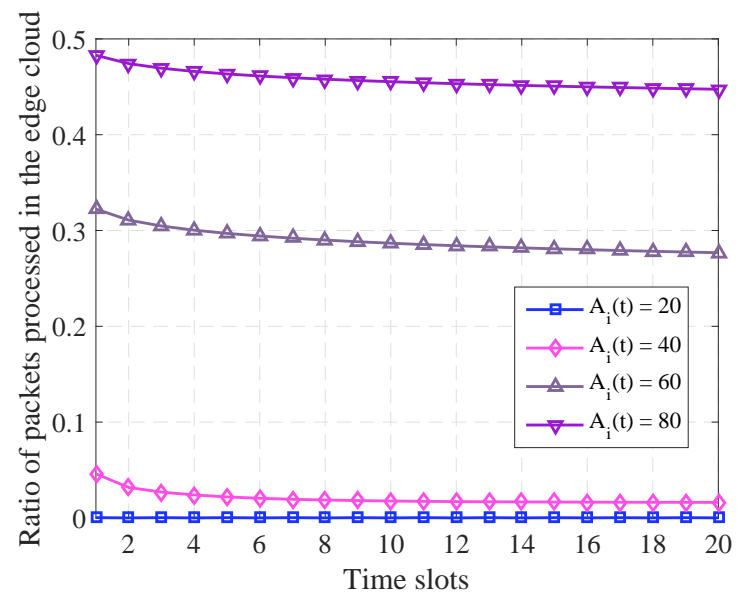

Fig. 8. The time evolution of the ratio of packets processed in the edge cloud (calculated by Eq. (43)) parameterized by different packet arrival rates.

and converges to the minimum value, when $V$ is sufficiently large. By contrast, the average queue length per user increases with $V$ and also converges, when $V$ is sufficiently large. It can be explained that when $V$ is sufficiently large, the optimal ratio of packets processed in the edge cloud tends to be a constant, so do the time-averaged power consumption and time-averaged queue length. These results quantify the tradeoff between the power consumption and execution delay, adjusted by the control parameter $V$.

The time evolution of the ratio of packets processed in the edge cloud $\eta_{i}(t)$ parameterized by the packets arrival rates is shown in Fig. 8, where we assume that there are $I=4$ FANETs and the packet arrival rates of the FANETs in each time slot is fixed to $\left[A_{1}(t), A_{2}(t), A_{3}(t), A_{4}(t)\right]=$ $[20,40,60,80], V$ is set to be 4000 . It is observed that our scheme tends to off-load more packets to the edge cloud for higher arrival rates. Specifically, all of the packets will be offloaded to the remote cloud when $A_{i}(t)=20$. Moreover, less packets will be off-loaded to the edge cloud, as time passes. The reason is that the queue length grows with the passage of time, thus more packets will be sent to the remote cloud to

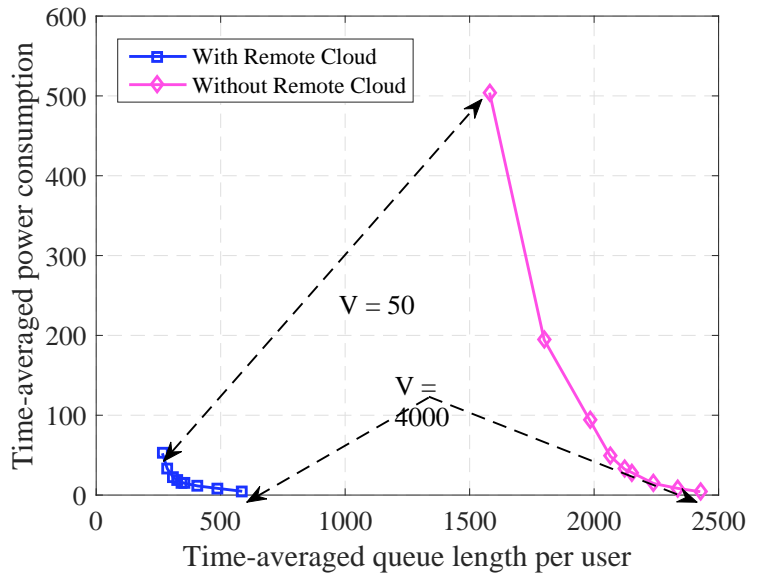

Fig. 9. The performance of average power consumption (calculated by Eq. (23)) and the system's queue length (calculated by Eq. (24)) both with and without the assistance of the remote cloud.

strike a power-vs-delay trade-off.

Fig. 9 depicts the comparison of the average power consumption versus the queue length of systems between the scenarios operating both with and without the remote cloud. Here we set $I=10$ and $V \in$ $\{50,100,200,300,400,500,1000,2000,4000\}$. It is shown that in general, the average power consumption decreases with the average queue length for both systems. Therefore, it is necessary to carefully select a suitable $V$ value to balance the power consumption vs execution delay. Moreover, the decayrate of the power consumption operating without without the remote cloud is more rapid than that of the system relying on the remote cloud, which indicates that the control parameter has more substantial influence on the system without the remote cloud. Additionally, more power is consumed by the system without the remote cloud, while leading to longer delays. This is because the edge cloud cannot stabilize its task buffers, due to its limited computational capacity, if it is not assisted by the remote cloud. In this case, Fig. 9 confirms the superiority of the heterogenous cloud system advocated.

\section{Conclusions}

In this paper, we studied the QoS-based network association problem of heterogenous cloud aided multi-UAV systems. We proposed an energy efficient gateway selection scheme for choosing the optimal UAV as the gateway in each time slot. Moreover, we jointly optimized the task scheduling and resource allocation in the heterogeneous cloud infrastructure. We formulated the problem as a power consumption minimization problem under specific system stability constraints. A power-vs-delay trade-off was struck as well as an optimal solution relying on Lyapunov optimization was designed. Our numerical results showed that our gateway selection scheme has a better energy consumption performance than other schemes. Furthermore, our proposed task scheduling and resource allocation scheme reaches the optimal solution within a few iterative rounds and yields an improved QoS performance. These results confirm that the heterogeneous 
cloud structure is beneficial for constructing high-performance multi-UAV systems.

\section{APPENDIX A}

\section{PROOF OF PROPOSITION 1}

Proof: The outage probability of data transmission between the gateway UAV $u$ and the AP can be expressed as:

$$
\begin{aligned}
f_{u} & =\operatorname{Pr}(\mathrm{SNR} \leq \beta) \\
& =\operatorname{Pr}\left(\frac{P_{u}\left(\xi_{1} f_{u}^{L o S}+\xi_{2} f_{u}^{N L o S}\right)^{2} d_{u}^{-\alpha}\left|\tilde{h}_{u}\right|^{2}}{N_{0}} \leq \beta\right) .
\end{aligned}
$$

Since $\left|\tilde{h}_{u}\right|$ follows Rician distribution according to Eq. (4), the distribution of $\left|\tilde{h}_{u}\right|^{2}$ is given by the non-central chi-square $\left(\chi^{2}\right)$ distribution [42], whose PDF can be expressed as:

$$
f_{\left|\tilde{h}_{u}\right|^{2}}(z)=\frac{1}{2 \sigma^{2}} \exp \left[-\frac{1}{2 \sigma^{2}}\left(z+A^{2}\right)\right] I_{0}\left(\frac{A}{\sigma^{2}} \sqrt{z}\right) .
$$

Hence, we have:

$$
\begin{aligned}
f_{u} & =\operatorname{Pr}\left(\left|\tilde{h}_{u}\right|^{2} \leq \Xi\right) \\
& =\int_{0}^{\Xi} \frac{1}{2 \sigma^{2}} \exp \left[-\frac{1}{2 \sigma^{2}}\left(z+A^{2}\right)\right] I_{0}\left(\frac{A}{\sigma^{2}} \sqrt{z}\right) d z,
\end{aligned}
$$

where $\Xi=\frac{N_{0} \beta d^{\alpha}}{P_{u}\left(\xi_{1} f_{u}^{L o S}+\xi_{2} f_{u}^{N L O S}\right)^{2}}$. Let $z=r^{2}$, then we obtain:

$$
\begin{aligned}
f_{u} & =\int_{0}^{\Xi} \frac{r}{\sigma^{2}} \exp \left[-\frac{1}{2 \sigma^{2}}\left(r^{2}+A^{2}\right)\right] I_{0}\left(\frac{A}{\sigma^{2}} r\right) d r \\
& =\int_{0}^{\Xi} \frac{r}{\sigma} \exp \left[-\frac{1}{2}\left(\left(\frac{r}{\sigma}\right)^{2}+\left(\frac{A}{\sigma}\right)^{2}\right)\right] I_{0}\left(\frac{A}{\sigma} \cdot \frac{r}{\sigma}\right) d\left(\frac{r}{\sigma}\right) \\
& =1-Q\left(\frac{A}{\sigma}, \Xi\right) .
\end{aligned}
$$

This completes the proof of Proposition 1.

\section{APPENDIX B \\ PROOF OF LEMMA 1}

Proof: We first use Eq. (7) to deduce the upper bound of the difference of the Lyapunov function between one time slot and the next time slot, which can be formulated as:

$$
\begin{aligned}
& L[Q(t+1)]-L[Q(t)] \\
& =\frac{1}{2} \sum_{i=1}^{I}\left[Q_{i}^{2}(t+1)-Q_{i}^{2}(t)\right] \\
& =\frac{1}{2} \sum_{i=1}^{I}\left[\left(\max \left\{Q_{i}(t)-\mu_{i}(t), 0\right\}+A_{e, i}^{2}(t)\right)^{2}-Q_{i}^{2}(t)\right] \\
& \leq \frac{1}{2} \sum_{i=1}^{I}\left[A_{e, i}^{2}(t)+\mu_{i}^{2}(t)-2 A_{e, i}(t) \mu_{i}(t)\right] \\
& \quad-\sum_{i=1}^{I}\left[Q_{i}(t)\left(\mu_{i}(t)-A_{e, i}(t)\right)\right] .
\end{aligned}
$$

For any $Q \geq 0, \mu \geq 0, A \geq 0$, we have:

$(\max \{Q-\mu, 0\}+A)^{2} \leq Q^{2}+\mu^{2}+A^{2}+2 A \mu-2 Q(\mu-A)$.

Thus, by computing the conditional Lyapunov function according to Eq. (29) and adding the penalty item of $V$. $E[P(t) \mid \boldsymbol{Q}(t)]$, we can achieve the upper bound in Lemma 1 . This completes the proof of Lemma 1.

\section{REFERENCES}

[1] J. Wang, C. Jiang, Z. Han, Y. Ren, R. G. Maunder, and L. Hanzo, "Taking drones to the next level: Cooperative distributed unmannedaerial-vehicular networks for small and mini drones," IEEE Vehicular Technology Magazine, vol. 12, no. 3, pp. 73-82, Jul. 2017.

[2] L. Gupta, R. Jain, and G. Vaszkun, "Survey of important issues in uav communication networks," IEEE Communications Surveys \& Tutorials, vol. 18, no. 2, pp. 1123-1152, Nov. 2016.

[3] M. Mozaffari, W. Saad, M. Bennis, and M. Debbah, "Unmanned aerial vehicle with underlaid device-to-device communications: Performance and tradeoffs," IEEE Transactions on Wireless Communications, vol. 15 , no. 6, pp. 3949-3963, Feb.

[4] Y. Zeng and R. Zhang, "Energy-efficient uav communication with trajectory optimization," IEEE Trans. Wireless Commun, vol. 16, no. 6, pp. 3747-3760, Jun. 2017.

[5] M. Mozaffari, W. Saad, M. Bennis, and M. Debbah, "Mobile unmanned aerial vehicles (uavs) for energy-efficient internet of things communications," IEEE Transactions on Wireless Communications, vol. 16, no. 11, pp. 7574-7589, Sep. 2017.

[6] Y. Chen, W. Feng, and G. Zheng, "Optimum placement of uav as relays," IEEE Communications Letters, vol. 22, no. 2, pp. 248-251, Nov. 2018.

[7] I. Bekmezci, O. K. Sahingoz, and Ş. Temel, "Flying ad-hoc networks (FANETs): A survey," Ad Hoc Networks, vol. 11, no. 3, pp. 1254-1270, May 2013.

[8] O. K. Sahingoz, "Networking models in flying ad-hoc networks (FANETs): Concepts and challenges," Journal of Intelligent \& Robotic Systems, vol. 74, no. 1-2, pp. 513-527, Apr. 2014.

[9] S. Rosati, K. Krużelecki, G. Heitz, D. Floreano, and B. Rimoldi, "Dynamic routing for flying ad hoc networks," IEEE Transactions on Vehicular Technology, vol. 65, no. 3, pp. 1690-1700, Mar. 2016.

[10] J. Wang, C. Jiang, Z. Ni, S. Guan, S. Yu, and Y. Ren, "Reliability of cloud controlled multi-UAV systems for on-demand services," in IEEE Global Communications Conference (GLOBECOM), Singapore, Dec. 2017, pp. 1-6.

[11] H. Su and X. Zhang, "Clustering-based multichannel MAC protocols for QoS provisionings over vehicular ad hoc networks," IEEE Transactions on Vehicular Technology, vol. 56, no. 6, pp. 3309-3323, Nov. 2007.

[12] S. Leng, Y. Zhang, H.-H. Chen, L. Zhang, and K. Liu, "A novel k-hop compound metric based clustering scheme for ad hoc wireless networks," IEEE Transactions on Wireless Communications, vol. 8, no. 1, pp. 367375, Feb. 2009.

[13] S. H. Bouk, I. Sasase, S. H. Ahmed, and N. Javaid, "Gateway discovery algorithm based on multiple QoS path parameters between mobile node and gateway node," Journal of Communications and Networks, vol. 14, no. 4, pp. 434-442, Aug. 2012.

[14] F. Hoffmann, D. Medina, and A. Wolisz, "Joint routing and scheduling in mobile aeronautical ad hoc networks," IEEE Transactions on Vehicular Technology, vol. 62, no. 6, pp. 2700-2712, Feb. 2013.

[15] G. Zhioua, N. Tabbane, H. Labiod, and S. Tabbane, "A fuzzy multimetric QoS-balancing gateway selection algorithm in a clustered VANET to LTE advanced hybrid cellular network," IEEE Transactions on Vehicular Technology, vol. 64, no. 2, pp. 804-817, Jun. 2015.

[16] M. Alawi, E. Sundararajan, R. Alsaqour, and M. Ismail, "QoS-enable gateway selection algorithm in heterogeneous vehicular network," in IEEE International Conference on Electrical Engineering and Informatics (ICEEI), Langkawi, Malaysia, Mar. 2017, pp. 1-6.

[17] M. Naeem, W. Ejaz, L. Karim, S. H. Ahmed, A. Anpalagan, M. Jo, and $\mathrm{H}$. Song, "Distributed gateway selection for M2M communication in cognitive 5G networks," IEEE Network, vol. 31, no. 6, pp. 94-100, Aug. 2017.

[18] F. Luo, C. Jiang, J. Du, J. Yuan, Y. Ren, S. Yu, and M. Guizani, "A distributed gateway selection algorithm for UAV networks," IEEE Transactions on Emerging Topics in Computing, vol. 3, no. 1, pp. 2233, Dec. 2015. 
[19] Y. C. Hu, M. Patel, D. Sabella, N. Sprecher, and V. Young, "Mobile edge computing-a key technology towards 5G," ETSI White Paper, vol. 11, no. 11 , pp. 1-16, Sep.

[20] F. Bonomi, R. Milito, J. Zhu, and S. Addepalli, "Fog computing and its role in the internet of things," in ACM Proceedings of the first edition of the MCC workshop on Mobile cloud computing, Helsinki, Finland, Aug. 2012, pp. 13-16.

[21] S. N. Shirazi, A. Gouglidis, A. Farshad, and D. Hutchison, "The extended cloud: Review and analysis of mobile edge computing and fog from a security and resilience perspective," IEEE Journal on Selected Areas in Communications, vol. 35, no. 11, pp. 2586-2595, Nov. 2017.

[22] S. Sardellitti, G. Scutari, and S. Barbarossa, "Joint optimization of radio and computational resources for multicell mobile-edge computing," IEEE Transactions on Signal and Information Processing over Networks, vol. 1, no. 2, pp. 89-103, Jun. 2015.

[23] Y. Mao, J. Zhang, S. Song, and K. B. Letaief, "Power-delay tradeoff in multi-user mobile-edge computing systems," in IEEE Global Communications Conference (GLOBECOM), Washington, DC, Feb. 2016, pp. $1-6$.

[24] Z. Jiang and S. Mao, "Energy delay trade-off in cloud offloading for mutli-core mobile devices," in IEEE 2015 Global Communications Conference (GLOBECOM), San Diego, CA, Dec. 2015, pp. 1-6.

[25] Y. Mao, J. Zhang, and K. B. Letaief, "Dynamic computation offloading for mobile-edge computing with energy harvesting devices," IEEE Journal on Selected Areas in Communications, vol. 34, no. 12, pp. 35903605, Sep. 2016.

[26] J. Liu, Y. Mao, J. Zhang, and K. B. Letaief, "Delay-optimal computation task scheduling for mobile-edge computing systems," in IEEE International Symposium on Information Theory (ISIT), Barcelona, Spain, Jul. 2016, pp. 1451-1455.

[27] X. Chen, L. Jiao, W. Li, and X. Fu, "Efficient multi-user computation offloading for mobile-edge cloud computing," IEEE/ACM Transactions on Networking, vol. 24, no. 5, pp. 2795-2808, Oct. 2016.

[28] F. Wang, J. Xu, X. Wang, and S. Cui, "Joint offloading and computing optimization in wireless powered mobile-edge computing systems," IEEE Transactions on Wireless Communications, vol. 17, no. 3, pp. 1784-1797, Dec. 2017.

[29] Z. Sanaei, S. Abolfazli, A. Gani, and R. Buyya, "Heterogeneity in mobile cloud computing: Taxonomy and open challenges," IEEE Communications Surveys \& Tutorials, vol. 16, no. 1, pp. 369-392, May. 2014.

[30] E. Gelenbe, R. Lent, and M. Douratsos, "Choosing a local or remote cloud," in The Second Symposium on Network Cloud Computing and Applications (NCCA), London, UK, Mar. 2012, pp. 25-30.

[31] Y. Wang and W. Shi, "Budget-driven scheduling algorithms for batches of MapReduce jobs in heterogeneous clouds," IEEE Transactions on Cloud Computing, vol. 2, no. 3, pp. 306-319, Apr. 2014.

[32] W. Wang, B. Liang, and B. Li, "Multi-resource fair allocation in heterogeneous cloud computing systems," IEEE Transactions on Parallel and Distributed Systems, vol. 26, no. 10, pp. 2822-2835, Oct. 2015.

[33] T. Zhao, S. Zhou, X. Guo, and Z. Niu, "Tasks scheduling and resource allocation in heterogeneous cloud for delay-bounded mobile edge computing," in IEEE International Conference on Communications (ICC), Paris, France, Jul. 2017, pp. 1-7.

[34] J. Du, L. Zhao, J. Feng, and X. Chu, "Computation offloading and resource allocation in mixed fog/cloud computing systems with min-max fairness guarantee," IEEE Transactions on Communications, vol. 66, no. 4, pp. 1594-1608, Dec. 2017.

[35] K. Gai, M. Qiu, and H. Zhao, "Energy-aware task assignment for mobile cyber-enabled applications in heterogeneous cloud computing," Journal of Parallel and Distributed Computing, vol. 111, pp. 126-135, Jan. 2018.

[36] M. J. Neely, "Stochastic network optimization with application to communication and queueing systems," Synthesis Lectures on Communication Networks, vol. 3, no. 1, pp. 1-211, 2010.

[37] A. Al-Hourani, S. Kandeepan, and S. Lardner, "Optimal lap altitude for maximum coverage," IEEE Wireless Communications Letters, vol. 3, no. 6, pp. 569-572, Jul. 2014.

[38] J. D. Little and S. C. Graves, "Little's law," in Building Intuition. Springer, 2008, pp. 81-100.

[39] G. Hooghiemstra and P. Van Mieghem, "Delay distributions on fixed internet paths," Delft University of Technology, Report, vol. 20011020, 2001.

[40] H. Chen, R. G. Maunder, and L. Hanzo, "A survey and tutorial on lowcomplexity turbo coding techniques and a holistic hybrid ARQ design example," IEEE Communications Surveys \& Tutorials, vol. 15, no. 4, pp. 1546-1566, Feb. 2013.

[41] S. Boyd and L. Vandenberghe, Convex optimization. Cambridge university press, 2004.
[42] J. Du, C. Jiang, Y. Qian, Z. Han, and Y. Ren, "Resource allocation with video traffic prediction in cloud-based space systems," IEEE Transactions on Multimedia, vol. 18, no. 5, pp. 820-830, Mar. 2016.

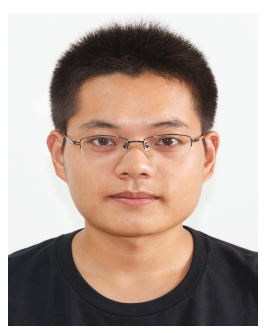

Ruiyang Duan (S'18) received his B.S. degree from Department of Electronic Engineering, Tsinghua University in 2016. He currently pursues his $\mathrm{PhD}$ degree in Department of Electronic Engineering, Tsinghua University, Beijing. His research areas include network resource allocation, UAV communications and mobile edge computing.

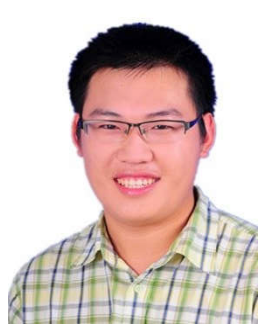

Jingjing Wang (S'14) received his B.S. degree in Electronic Information Engineering from Dalian University of Technology in 2014 with the highest honor. He currently works for his $\mathrm{PhD}$ degree in Department of Electronic Engineering, Tsinghua University, Beijing. From 2017 to 2018, he has been a joint $\mathrm{PhD}$ student in Next Generation Wireless Group chaired by Prof. Lajos Hanzo, University of Southampton, UK. His research interests include the resource allocation and network association, learning theory aided modeling, analysis and signal processing, as well as information diffusion theory for mobile wireless networks. He received Tsinghua GuangHua Scholarship Award in 2016 and graduate China National Scholarship Award in 2017.

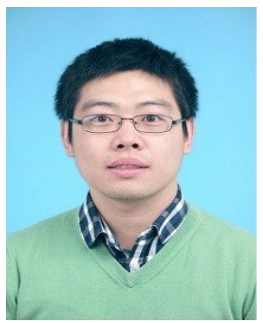

Chunxiao Jiang (S'09-M'13-SM'15) received the B.S. degree from Beihang University, Beijing in 2008 and the Ph.D. degree in electronic engineering from Tsinghua University, Beijing in 2013, both with the highest honors. From 2011 to 2012, he visited University of Maryland, College Park as a joint PhD supported by China Scholarship Council. From 2013 to 2016, he was a postdoc with Tsinghua University, during which he visited University of Maryland, College Park and University of Southampton. Since July 2016, he became an assistant professor in Tsinghua Space Center, Tsinghua University. His research interests include space networks and heterogeneous networks. Dr. Jiang is the recipient of the Best Paper Award from IEEE GLOBECOM in 2013, the Best Student Paper Award from IEEE GlobalSIP in 2015, IEEE Communications Society Young Author Best Paper Award in 2017, the Best Paper Award IWCMC in 2017, the Best Journal Paper Award of IEEE ComSoc Technical Committee on Communications Systems Integration and Modeling 2018.

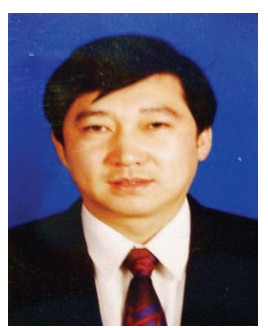

Yong Ren (SM'16) received his B.S, M.S and $\mathrm{Ph} . \mathrm{D}$. degrees in electronic engineering from Harbin Institute of Technology, China, in 1984, 1987, and 1994 , respectively. He worked as a post doctor at Department of Electronics Engineering, Tsinghua University, China from 1995 to 1997. Now he is a professor of Department of Electronics Engineering and the director of the Complexity Engineered Systems Lab in Tsinghua University. He holds 12 patents, and has authored or co-authored more than 100 technical papers in the behavior of computer network, P2P network and cognitive networks. He has serves as a reviewer of IEICE Transactions on Communications, Digital Signal Processing, Chinese Physics Letters, Chinese Journal of Electronics, Chinese Journal of Computer Science and Technology, Chinese Journal of Aeronautics and so on. His current research interests include complex systems theory and its applications to the optimization and information sharing of the Internet, Internet of Things and ubiquitous network, cognitive networks and Cyber-Physical Systems. 


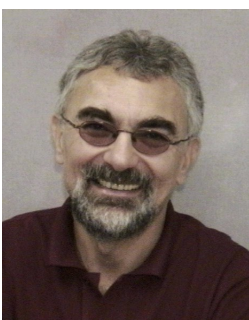

Lajos Hanzo (http://www-mobile.ecs.soton.ac.uk) FREng, F'04, FIET, Fellow of EURASIP, received his 5-year degree in electronics in 1976 and his doctorate in 1983 from the Technical University of Budapest. In 2009 he was awarded an honorary doctorate by the Technical University of Budapest and in 2015 by the University of Edinburgh. In 2016 he was admitted to the Hungarian Academy of Science. During his 40-year career in telecommunications he has held various research and academic posts in Hungary, Germany and the UK. Since 1986 he has been with the School of Electronics and Computer Science, University of Southampton, UK, where he holds the chair in telecommunications. He has successfully supervised $119 \mathrm{PhD}$ students, co-authored 18 John Wiley/IEEE Press books on mobile radio communications totalling in excess of 10000 pages, published $1800+$ research contributions at IEEE Xplore, acted both as TPC and General Chair of IEEE conferences, presented keynote lectures and has been awarded a number of distinctions. Currently he is directing a 60 -strong academic research team, working on a range of research projects in the field of wireless multimedia communications sponsored by industry, the Engineering and Physical Sciences Research Council (EPSRC) UK, the European Research Council's Advanced Fellow Grant and the Royal Society's Wolfson Research Merit Award. He is an enthusiastic supporter of industrial and academic liaison and he offers a range of industrial courses. He is also a Governor of the IEEE ComSoc and VTS. During 2008 - 2012 he was the Editor-in-Chief of the IEEE Press and a Chaired Professor also at Tsinghua University, Beijing. For further information on research in progress and associated publications please refer to http://www-mobile.ecs.soton.ac.uk. 